\title{
Contour Encoding Based on Extraction of Key Points Using Wavelet Transform
}

\author{
Vishnu Makkapati \\ Honeywell Technology Solutions Lab \\ 151/1, Doraisanipalya, Bannerghatta Road \\ Bangalore - 560 076, India \\ vishnu.makkapati@honeywell.com
}

\author{
Pravas Mahapatra \\ Department of Aerospace Engineering \\ Indian Institute of Science \\ Bangalore - 560 012, India \\ pravas@aero.iisc.ernet.in
}

\begin{abstract}
In many situations it is convenient to represent pictorial data in the form of contours. It may become necessary to compress such contour data for efficient storage and transmission. We present here a technique for achieving very high levels of compression of 2-D contours. The goal here is to represent each contour using a discrete set of representative points known as key points. A novel method of extracting the key points using wavelet transform is presented. The scheme exploits the properties of the high frequency coefficients to identify these points. Local peaks in the magnitude plot of high frequency coefficients are designated as key points and are identified using an efficient algorithm. The performance of the scheme is evaluated using multiple actual contours derived from weather radar reflectivity fields.
\end{abstract}

\section{Introduction}

Encoding of contours finds applications in many diverse areas such as generalization of maritime lines, representation of object shapes, and compression of weather radar data. Recently, contour encoding has attracted increasing interest due to region-based image and video coding methods [6]. Many lossless and lossy approaches have been proposed for contour encoding.

Lossless encoding methods such as chain coding [4] generally yield only modest degrees of compression. Further, with incremental coding schemes (which also include chain coding) any local error propagates through the remaining part of the contour. This had led to the development of lossy encoding schemes that offer the potential of high levels of compression with very little loss of information.

Polynomial and parametric approximations are suitable lossy encoding methods for relatively smooth contours, but are not accurate in the presence of rapid random undulations. Polygonal approximation is a commonly used

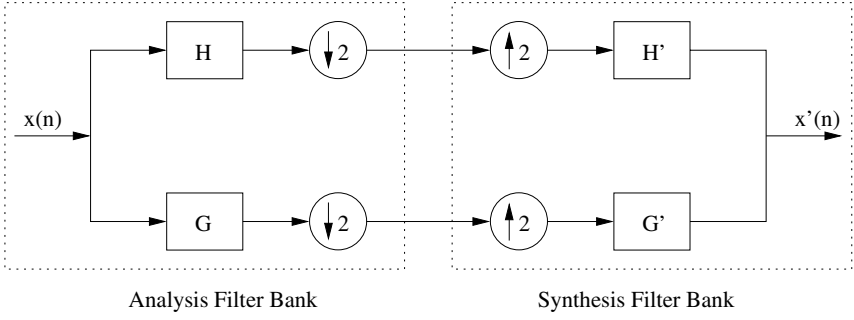

Figure 1. 1-D wavelet analysis and synthesis filter banks

method as it offers a natural representation and the bit rate can be adjusted based on the degree of approximation. In this method, a contour is approximated using straight line segments, the end points of which are designated as key points (or vertices) which are transmitted to the receiving end. The methods proposed by Douglas and Peucker [2], Teh and Chin [7], Gertz and Grappel [5], and Burdon [1] are examples of polygonal coding methods.

In polygonal approximation of contours, the choice of the key points is critical to the achievable compression and the fidelity of reconstruction. In this paper we propose a novel method of choosing key points for rapidly undulating contours using a discrete wavelet transform (DWT) approach. The scheme analyses each contour in terms of its low and high frequency contents and exploits the properties of the high frequency coefficients to identify the key points. In particular, the local peaks in the magnitude plot of high frequency coefficients are designated as key points and are identified using an efficient algorithm.

\section{Discrete Wavelet Transform}

The framework of a one-dimensional DWT is shown in Fig. 1 where blocks $H$ and $G\left(H^{\prime}\right.$ and $\left.G^{\prime}\right)$ represent low pass and high pass analysis (synthesis) filters respectively. Perfect reconstruction is achieved if the filters $H, G, H^{\prime}$, 


\begin{tabular}{|l|l|l|l|}
\hline $\mathrm{L}_{3}$ & $\mathrm{H}_{3}$ & $\mathrm{H}_{2}$ & $\mathrm{H}_{1}$ \\
\hline
\end{tabular}

\section{Figure 2. Subbands for a three-level one- dimensional DWT}

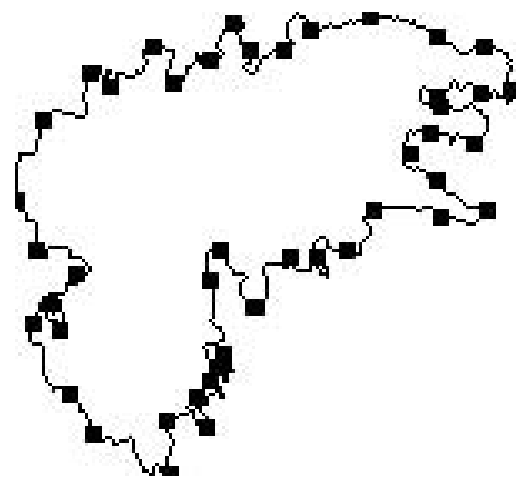

Figure 3. Example contour and its key points (square dots) obtained by DWT analysis

and $G^{\prime}$ satisfy

$$
\begin{aligned}
H^{\prime}(z) H\left(z^{-1}\right)+G^{\prime}(z) G\left(z^{-1}\right) & =2 \\
H^{\prime}(z) H\left(-z^{-1}\right)+G^{\prime}(z) G\left(-z^{-1}\right) & =0
\end{aligned}
$$

Under these conditions the aliasing caused by down sampling will be canceled at the synthesis.

Multiple levels or scales of DWT can be obtained by repeating the filtering and decimation operations on the low frequency coefficients only. The subband labeling scheme for a three-level, 1-D DWT is shown in Fig. 2. Labels $L$ and $H$ denote low frequency and high frequency coefficients respectively while the subscript denotes the resolution level. The detail (high frequency) coefficients $H_{i}, 1 \leq i \leq 3$ describe the information lost while going from an approximation with resolution $2^{i-1}$ to a coarser approximation with resolution $2^{i}$.

\section{Proposed Scheme}

A 2-D contour can be specified using the coordinates of its points $\left(x_{i}, y_{i}\right), 1 \leq i \leq N$ where $N$ denotes the number of points on the contour and satisfies the conditions (1) $\left|x_{i}-x_{i+1}\right| \leq 1$ and $\left|y_{i}-y_{i+1}\right| \leq 1$, and (2) $\left(x_{N+i}, y_{N+i}\right)=\left(x_{i}, y_{i}\right)$. Circular convolution can be employed to convolve a contour with a filter since contours are inherently 'circular' or closed in nature.

The proposed scheme analyzes a contour using DWT. Since circular convolution is used, the problem of signal extension at the boundary does not arise and a wide variety

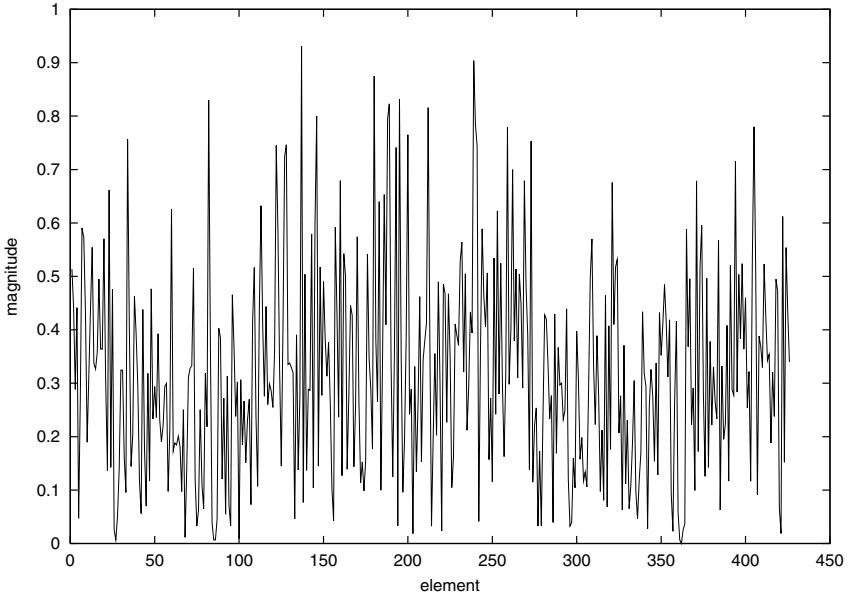

Figure 4. High frequency subband coefficients for 1-level of DWT analysis

of DWT filters can be used. The low pass filter $H$ smooths a contour while the high pass filter $G$ captures the local variations of the contour. The magnitude of a coefficient in the high frequency subband, defined as $\left.\sqrt{(} x_{i}^{h^{2}}+y_{i}^{h^{2}}\right)$, represents the variation along both $\mathrm{x}$ - and $\mathrm{y}$ - directions.

The proposed scheme exploits the property of the high frequency coefficient magnitude to extract key points of the contour. The magnitude of a coefficient at a given point is high when the shape of the contour changes abruptly at that point. In smooth segments of a contour the magnitude of the coefficients will be small. A plot of the magnitude of the coefficients thus helps in determining the key points.

An example contour and the plot of the magnitude of its high frequency coefficients at first level of DWT analysis are shown in Figs. 3 and 4 respectively. The magnitude drops to zero at certain points that lie at the midpoint of absolutely smooth segments of the contour. The peaks represent the points where the shape of the contour varies rapidly and hence these points can be designated as key points. The points adjacent to peaks also have significant magnitude.

The goal here is to extract the local peaks in the magnitude plot of the high-frequency coefficient. Several methods can be used to determine these local peaks. One approach could be to consider those points corresponding to a designated number of highest peak values as the key points. However, all the key points may not be captured if the magnitude of their high frequency coefficients varies over a larger range.

Another approach may be to divide a contour into uniform segments and take the point with maximum magnitude in each segment as a key point. This scheme can be scaled to any desired compression ratio by varying the length of the segments. However, this scheme depends on the choice (length and location) of these segments and may not per- 


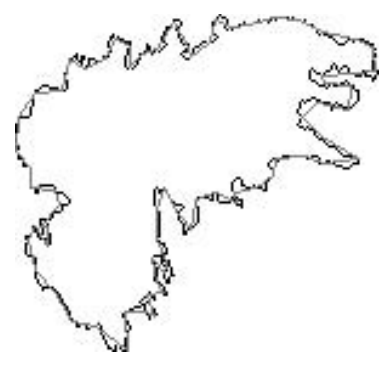

(a)

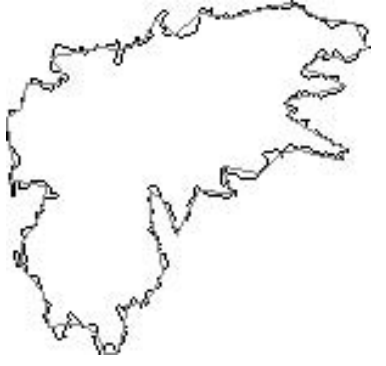

(b)

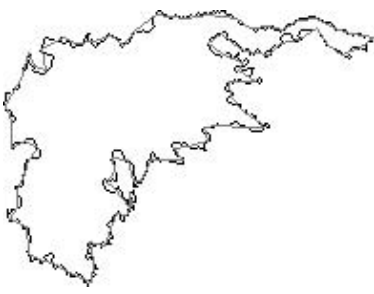

(c)

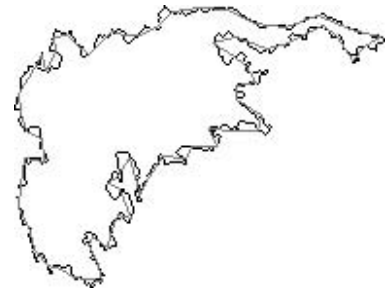

(d)

\section{Figure 5. Four original weather radar reflectivity contours (black) and their corresponding recon- structed contours (grey)}

form well when individual segments harbor multiple points with comparable maxima. An adaptive contour segment choice is likely to lead to better results.

In this paper, a threshold based method is proposed to locate key points. A maximum lying between two points whose magnitudes fall below a certain threshold is designated as a key point. However, this scheme introduces a large number of key points when the magnitude crosses the threshold frequently. To overcome this drawback, we stipulate a minimum separation between valid key points. Further, a key point may be added midway between two successive key points if their arc distance exceeds a certain length. It may be desirable to make these lengths a fraction of the contour length (perimeter) to make them independent of the length of the contour.

If a magnitude peak occurs at the $i^{\text {th }}$ point in the high frequency subband, the scheme considers $(2 i+1)^{t h}$ point and $(2 i)^{\text {th }}$ point to be a key point when the high pass filter is centered on odd and even points respectively. Either of the two modes of convolution (odd or even centered) may be employed and the key points extracted may deviate by one point if the other mode was a better choice. This one-point deviation does not significantly degrade the performance of the scheme. If the computational complexity is not an issue, both the modes can be tried and the better one can be chosen.

A multitude of wavelet filters are available and the choice of these filters depends on the application. In the current application, short wavelet filters may result in less significant magnitude peaks as variations over a shorter segment of a contour are captured. Hence, it may be ideal to select longer tap wavelet filters with good energy compaction property. The method proposed in this paper uses biorthogonal modified Coiflet filters [8].

The square dots in Fig. 3 visually depict that the proposed scheme extracts most of local peaks of the contour as key points. The proposed scheme is extremely memory efficient since DWT permits in-place calculations. Minimal computational resources are required since magnitude is calculated only for half the points in a contour, and faster implementations of DWT exist. The local peak search process can be performed with $O(n)$ complexity and is very efficient.

The fidelity of the scheme can be adjusted against compression ratio (which depends on the number of key points) by optimal choice of the threshold and the minimum/maximum segment length between successive key points. The choice of higher thresholds will result in a smaller number of key points and vice versa. A preferred choice would be to retain as many local peaks as possible by choosing a lower threshold and then eliminate the less critical key points by using a larger minimum segment length. At the receiving end, the contour is reconstructed by joining successive key points using Bresenham's line drawing algorithm [3].

\section{Performance Evaluation}

In the proposed scheme the compression ratio, expressed as the ratio of number of contour points to the key points, quantifies the saving in data volume. The performance of contour encoding methods can be qualitatively assessed by visual comparison and quantitatively evaluated using a criterion such as root-mean-square-error (RMSE). However, a metric such as RMSE captures the gross deviation of a reconstructed contour from its original and does not depict the distribution of the deviation at each point.

Geometric distortion offers a very stringent measure where the distance between a point on the original contour and its nearest point on the reconstructed contour must not exceed a certain threshold. Hence, a histogram of the deviations of all the points is also included to judge the performance of the scheme. 


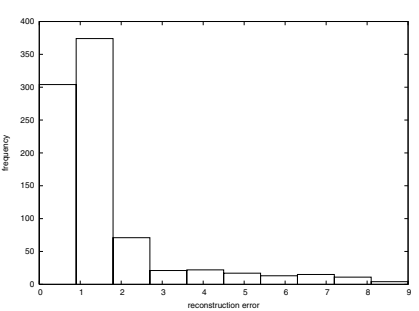

(a)

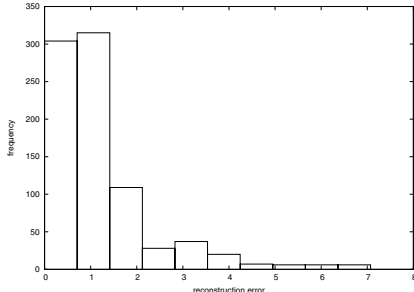

(b)

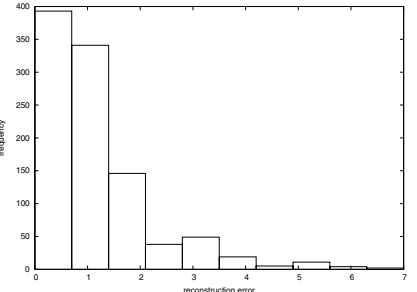

(c)

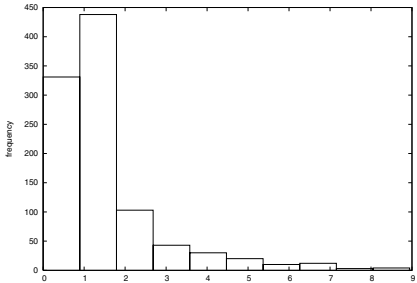

(d)

Figure 6. Histogram of reconstruction errors for the contours shown in Fig. 5

RMSE calculation is non-trivial since there is no one-toone correspondence between the points in the original and reconstructed contours. Further, the number of points in the original and reconstructed contours may differ. A brute force search for the nearest point on the reconstructed contour for each point on an original contour incurs significant computational cost. The proposed scheme uses a simple and efficient method to calculate RMSE.

The proposed scheme first finds a point on the reconstructed contour with minimum deviation (ideally 0 ) from a point on the original contour. The search for nearest point for each of the remaining points on the original contour is carried out within a certain neighborhood on either side of the previous nearest point on the reconstructed contour. This neighborhood can be considered to be a fraction of the original contour length.

We apply the method described above to a set of four contours obtained by thresholding weather radar reflectivity data. A comparison of original and reconstructed contours in Fig. 5 shows that the proposed scheme represents all the contours faithfully. In Table 1 The RMSE between original and reconstructed contours for the DWT method is compared with those obtained from the methods of Burdon [1] and Gertz and Grappel [5], using as basis a common compression ratio as close to 12 as possible. These results show that the accuracy of the DWT encoding is better than Burdon's scheme for all the 4 data sets, and it surpasses Gertz and Grappel's method in one case.

Table 1. RMS contour retrieval error in pixels

\begin{tabular}{|c|c|c|c|c|}
\hline Contour & $\begin{array}{c}\text { Compression } \\
\text { ratio }\end{array}$ & DWT & Burdon & $\begin{array}{c}\text { Gertz \& } \\
\text { Grappel }\end{array}$ \\
\hline $\mathrm{a}$ & 12.0 & 1.6448 & 1.7256 & 2.1035 \\
\hline $\mathrm{b}$ & 12.1 & 1.7248 & 1.8337 & 1.6130 \\
\hline $\mathrm{c}$ & 11.7 & 1.5728 & 1.8397 & 1.5147 \\
\hline $\mathrm{d}$ & 13.8 & 2.0339 & 2.1137 & 1.9041 \\
\hline
\end{tabular}

The histograms (Fig. 6) of reconstruction errors for all the points in the original contours in Fig. 5 show that the range of deviation of individual points is from 0 to 8 pixels, but over $65 \%$ of the reconstructed points remain within one pixel from their original location.

\section{Conclusions}

A novel wavelet based polygonal approximation method for encoding of contours is presented. This scheme captures all the significant key points by searching for local peaks in the plot of magnitude of high frequency coefficients. Results presented for various contours show that the scheme performs very well for complex and randomly undulating contour shapes. The performance of the scheme using various wavelet filters can be further evaluated.

\section{References}

[1] D. Burdon. System and method for the adaptive mapping of matrix data to set of polygons. U.S. Patent 6614425, September 2003.

[2] D. H. Douglas and T. K. Peucker. Algorithms for the reduction of the number of points required to represent a line or its carricature. The Canadian Cartographer, 10(2):112 - 122, 1973.

[3] J. D. Foley, A. V. Dam, S. K. Feiner, and J. F. Hughes. Computer Graphics: Principles and Practice in C. AddisonWesley, Reading, MA, second edition, 1995.

[4] H. Freeman. On encoding arbitrary geometric configurations. IRE Transactions on Electronic Computers, 10:260 - 268, 1961.

[5] J. L. Gertz and R. D. Grappel. Storage and transmission of compressed weather maps and the like. U.S. Patent 5363107, November 1994.

[6] A. Katsaggelos, L. Kondi, F. Meier, J. Ostermann, and G. Schuster. Mpeg-4 and rate-distortion-based shape-coding techniques. Proceedings of the IEEE, 86(6), June 1998.

[7] C. Teh and R. Chin. On the detection of dominant points on digital curves. IEEE Transactions on Pattern Analysis and Machine Intelligence, 11(8), August 1989.

[8] L. Winger and A. Venetsanopoulos. Biorthogonal modified coiflet filters for image compression. In IEEE International Conference on Acoustics, Speech, and Signal Processing, volume 5, pages 2681 - 2684, Seattle, May 1998. 